Volume 9, No.1.4, 2020

International Journal of Advanced Trends in Computer Science and Engineering

Available Online at http://www.warse.org/IJATCSE/static/pdf/file/ijatcse9691.42020.pdf

https://doi.org/10.30534/ijatcse/2020/9691.42020

\title{
Dual-Arm Robot with Mobile Robot Platform with Master-Slave Configuration for Teleoperation Application
}

\author{
Mohd Hairi Mohd Zaman, Mohd Faisal Ibrahim, Nasharuddin Zainal, Muhammad Fikri Nasir \\ Department of Electrical, Electronic and Systems Engineering, Faculty of Engineering and Built Environment, \\ Universiti Kebangsaan Malaysia, 43600 Bangi, Selangor, Malaysia \\ hairizaman@ukm.edu.my, faisal.ibrahim@ukm.edu.my, nasharuddin.zainal@ukm.edu.my, \\ mfn2704@gmail.com
}

\begin{abstract}
Teleoperation applications nowadays use the mobile robot platform with attached robot arms and controlled remotely. For safety-critical applications such as health screening and swab testing in the medical field, human operators need to control the robot arm accurately. A standard remote control method like joystick requires high skilled personnel to operate the robot. Therefore, this work proposed a dual robot arm system with one of the arms is attached to a mobile robot platform. Meanwhile, the human operator manually moves another robot arm. At the same time, the robot arm on the mobile platform mimics the robot arm movement controlled by the human. This teleoperation procedure uses the master-slave configuration, which the first and second robot arm act as the master and slave, respectively. Both simulation and hardware testing validate the master-slave teleoperation performance.
\end{abstract}

Key words : Dual-arm robot, mobile robot, manipulator, teleoperation, master-slave.

\section{INTRODUCTION}

The teleoperation robot system has received much attention in recent years due to its mobility and safety properties, which offer essential benefits[1], [2]. Typically, this type of robot system consists of a mobile robot system, controlled remotely by the human for executing specific dangerous and safety-critical tasks[3]-[5]. It has many possible uses, for example,in the medical field, such as for health screening and swab tests, especially during pandemics like influenza and COVID-19 outbreak. Currently, the swab test procedure involves taking the human sample either through the nose or throat [6], [7]. However, this swab test procedure exposes the frontline staff, who are the medical doctors and nurses, to the high risk of infection from the individual who has been infected by the virus because they need to have close contact during the procedure. One of the ways to prevent close contact between the frontline staff and patients is to use the teleoperation robot system.
Teleoperation robots have been used to assist the medical staff in hospitals for disinfection purposes, drug and food delivery to the warded patients, and also as the communication medium between the doctors and patients [8], [9]. However, the teleoperation robot for the swab test and health screening application is not widely used and utilizes only one robot arm [10], [11]. Typically, a highly-skill human remotely controls the teleoperation robot, either using a regular joystick or other remote control devices. However, the robot needs to accurately move its end-effector close to the human for a specific medical procedure like the swab test. This procedure requires high skill and accurate positioning. Therefore, a dual-arm robot system has great potential in teleoperation applications[12]. In general, various industrial routine tasks use the dual-arm robot [13]-[20].In the medical field, robots assist highly-skill and critical medical procedures [14], [21]-[23].

The present paper presents a teleoperation robot system to fulfill the need. The proposed system has a dual-arm robot and a mobile robot platform. The mobile robot platform has one of the robot arms, while a human operator manually moves another robot arm in the control room during the critical task. The robot arm on the mobile robot platform mimics any movement of the robot arm in the control room. This combination of robot arm formed an improved teleoperation application in which increases the flexibility and accuracy with minimum human operator skill.

\section{METHODOLOGY}

The proposed teleoperation robot system in this work consists of two sets of robot arms and a mobile robot platform. The selected robot arm model is OpenMANIPULATOR-X from Robotis[24]. Meanwhile, the mobile robot model is TurtleBot3, also from Robotis[25]. Both robot arm and mobile robot are low-cost devices and suitable for prototype purposes. Furthermore, all design files are open-source and available online. Besides, all simulation and control software usedRobot Operating System (ROS), which is free and an open-source middleware software platform[26], [27]. ROS is a popular software framework in developing robots, which support various operating systems such as Linux, Mac OS, or 
Microsoft Windows. ROS uses a lot of hardware abstraction and has advanced simulation capabilities with tools for testing and visualization.

Figure 1 shows the block diagram of the dual-arm robot system with a mobile robot platform, developed in this work. In this work, there were two robot arms, namely Master Robot Arm (MRA) and Slave Robot Arm (SRA), model OpenMANIPULATOR-X. MRA was mounted in a fixed location, such as in the remote control room. For example, the frontline staff, who is also the human operator, during the swab test procedure, will be in this remote control room. Meanwhile, SRA was mounted on top of the mobile robot, model TurtleBot3. The human operator was able to remotely control SRA to move near the task location, such as near the patient for swab test purpose, in the patient room or ward in the hospital.

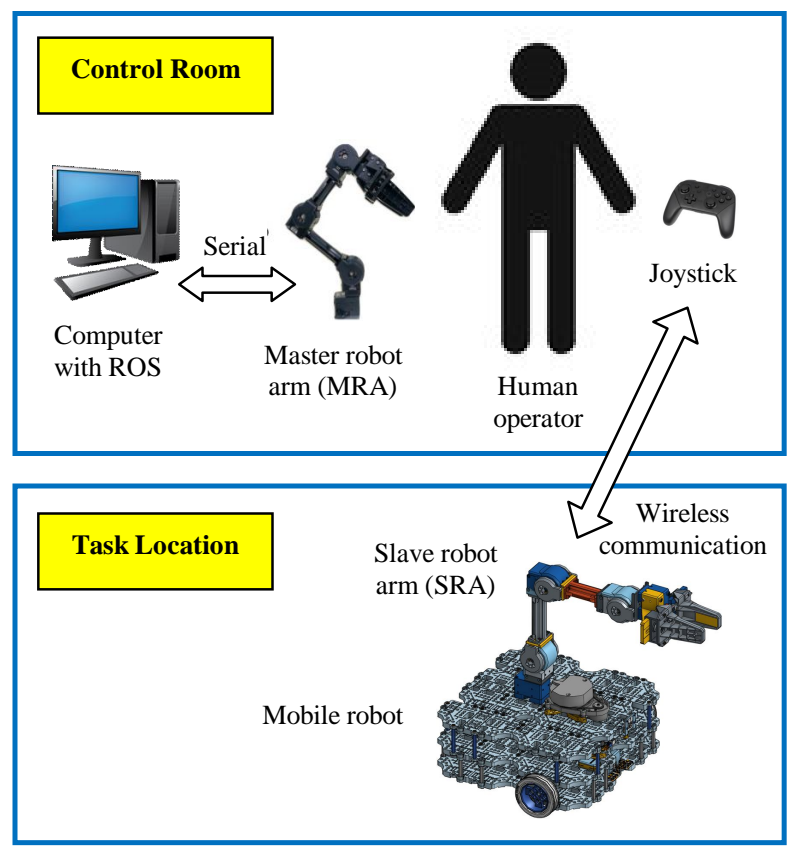

Figure1: Dual-arm robot system with a mobile robot platform

Furthermore, Figure 2 depicts the operation flowchart of the dual-arm robot system with a mobile robot platform. Firstly, the human operator remotely controlled the SRA movement from the initial location at the control room to the task location using a regular joystick, model RC100 from Robotis. The joystick used wireless communication, i.e., via Bluetooth. Then, after arriving at the task location, the human operator manually moved and guided MRA using hands to mimic the required task, such as swab test. At the same time, SRA followed any MRA movement in real-time. All MRA joint angle information was transferred to SRA through the RS485 communication protocol. Finally, after the task has been completed, the human operator controlled the SRA to go back to its home location at the control room.

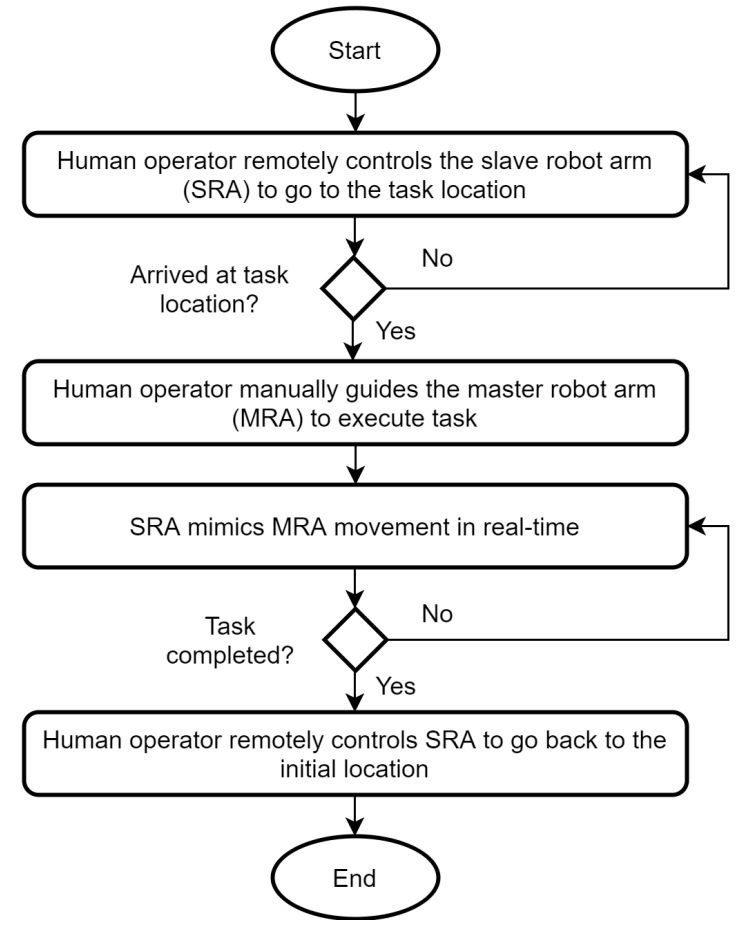

Figure 2: Operation flowchart of the dual-arm robot system with a mobile robot platform

\section{RESULTS AND DISCUSSION}

Figure 3 shows an example of an environment where the proposed dual-arm robot with a mobile robot platform can be used. This environment was simulated using Gazebo and ROS in Ubuntu 16 operating system. There are two separate rooms, which are the remote control room and patient room.

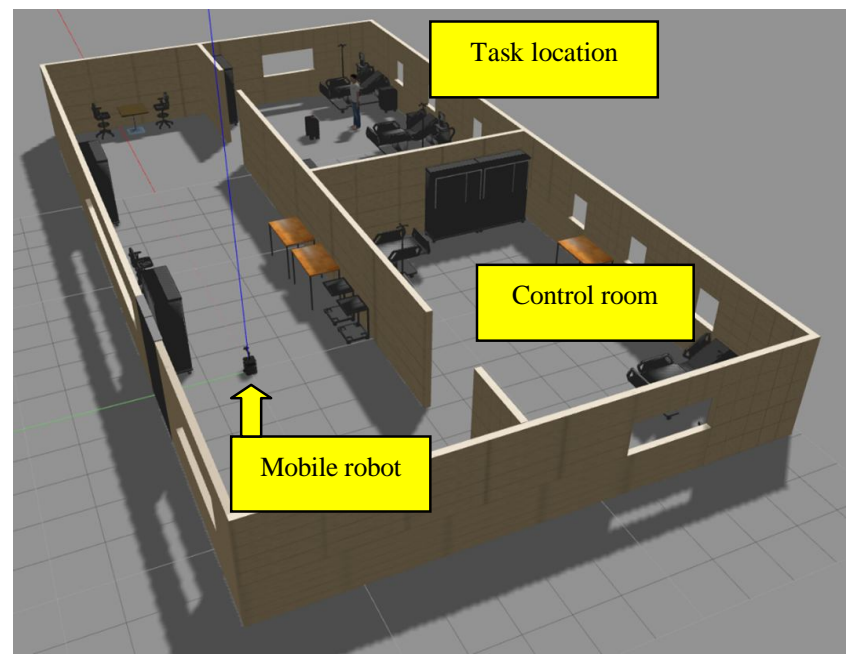

Figure 3: Example of environment for the dual-arm robot system with a mobile robot platform 
Meanwhile, Figure 4 depicts the prototype of the proposed robot system in this work using real hardware. The real system consists of a computer, where resides ROS and simulation software, two robot arms (MRA and SRA), a remote control joystick to control SRA, a mobile robot, and the RS485 communication board. Besides, Figure 5 shows the comparison between the simulation and real hardware implementation of MRA. The result shows that the real robot arm movement is the same as the simulated robot arm. Furthermore, SRA able to mimic the MRA movement in real-time, as shown in Figure 6.

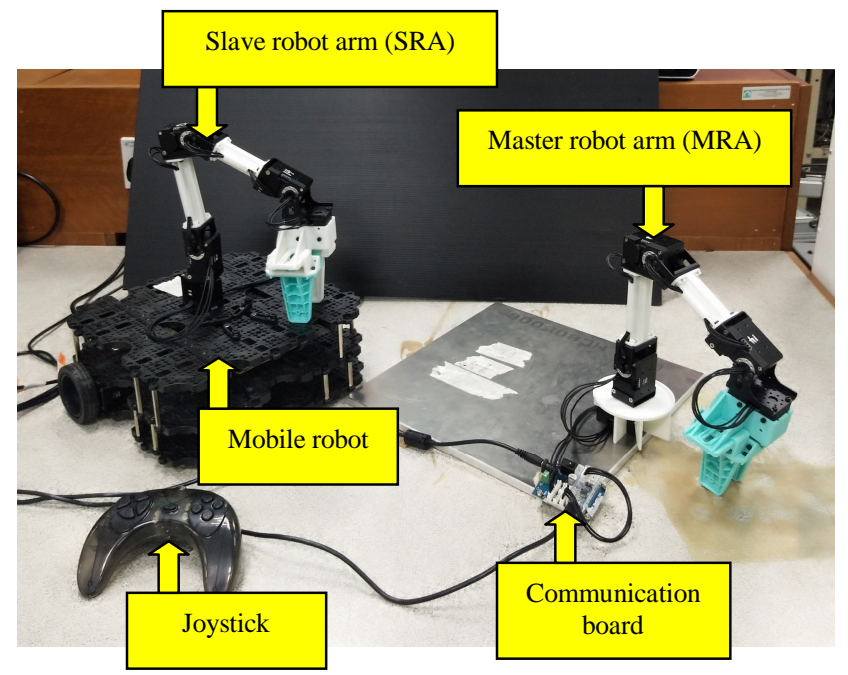

Figure 4: Real hardware implementation of the dual-arm robot system with a mobile robot platform

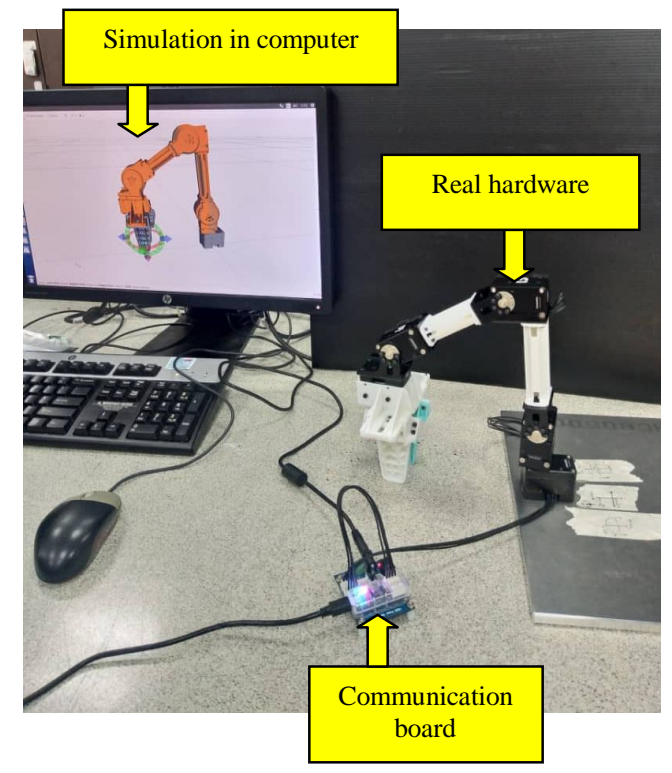

Figure 5: Both simulated and real hardware (master robot arm) movement of the robot arm are the same

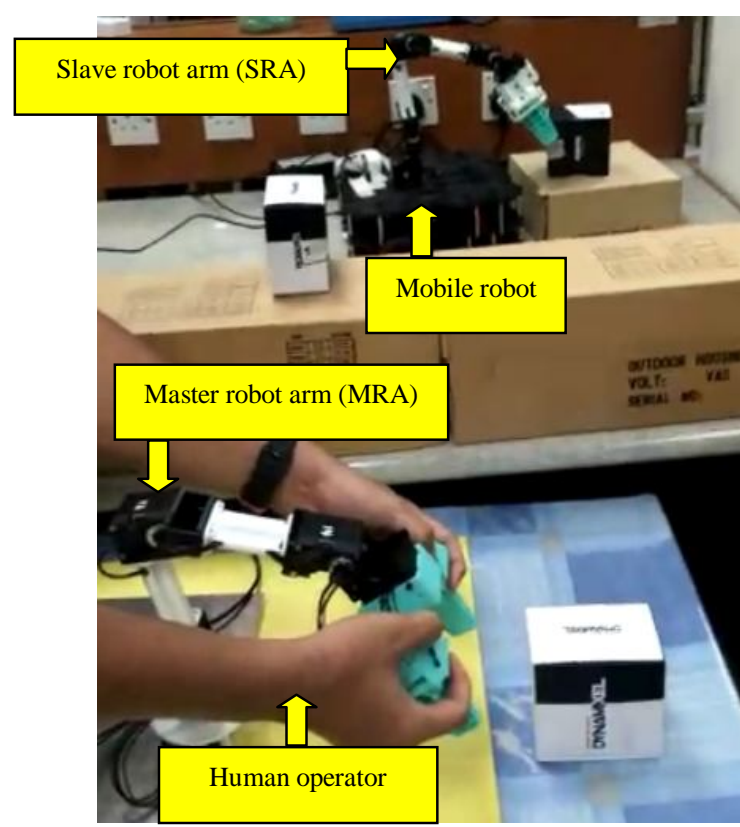

Figure 6: SRA mimics MRA movement in real-time

\section{CONCLUSION}

This work developed a working prototype of a dual-arm robot system with a mobile robot platform. Simulation and actual robot hardware testing outcomes show that the whole system can operate well with further improvement. This promising outcome acts as a groundwork for the real case implementation in the future, for example, integrating a vision-based system using a camera for autonomous and closed-loop operation of the robot system.

\section{ACKNOWLEDGEMENT}

This work was financially supported by Universiti Kebangsaan Malaysia under the Research University Grant no. GUP-2018-103 and Ministry of Higher Education Malaysia under the Fundamental Research Grant Scheme no. FRGS/1/2018/TK04/UKM/02/16.

\section{REFERENCES}

1. A. Ollero et al.The AEROARMS Project: Aerial robots with advanced manipulation capabilities for inspection and maintenance, IEEE Robotics and Automation Magazine, vol. 25, no. 4, pp. 12-23, 2018.

2. M. Pecka, K. Zimmermann, M. Reinstein, and T. Svoboda.Controlling robot morphology from incomplete measurements, IEEE Trans. Ind. Electron., vol. 64, no. 2, pp. 1773-1782, 2017. https://doi.org/10.1109/TIE.2016.2580125

3. H. M. Agrawal and S. G. Mundada.ORBOID: Robotic VR based application for remote regions, Int. J. $A d v$. Trends Comput. Sci. Eng., vol. 9, no. 1, pp. 845-851, 2020.

https://doi.org/10.30534/ijatcse/2020/121912020 
4. E. Yano, L. S. Reyes, P. R. Castro, J. J. Tarroza, and A. James.Prototype of a compact assistant surveillance robot for search and rescue operations, Int. $J . A d v$. Trends Comput. Sci. Eng., vol. 9, no. 1.1, pp. 64-68, 2020.

https://doi.org/10.30534/ijatcse/2020/1291.12020

5. E. B. Panganiban.Automated hazardous gas detecting robot using wireless sensor networks with GSM-SMS alert and fire control system for households, Int. J. Adv. Trends Comput. Sci. Eng., vol. 8, no. 3, pp. 8040-809, 2019.

https://doi.org/10.30534/ijatcse/2019/72832019

6. Centers for Disease Control and Prevention.Interim Guidelines for Collecting, Handling, and Testing Clinical Specimens from Persons for Coronavirus Disease 2019 (COVID-19), 2020. [Online]. Available: https://www.cdc.gov/coronavirus/2019-ncov/lab/guideli nes-clinical-specimens.html.

7. T. Singhal.A review of coronavirus disease-2019 (COVID-19), Indian J. Pediatr., vol. 87, no. 4, pp. 281-286, 2020.

8. G.-Z. Yang et al.Combating COVID-19 - The role of robotics in managing public health and infectious diseases, Sci. Robot., vol. 5, no. 40, p. eabb5589, 2020.

9. A. Rodić, D. Pisla, and H. Bleuler, Eds. New Trends in Medical and Service Robots: Challenges and Solutions,Switzerland: Springer, 2014, pp. 177-192

10. B. Farmer.Engineers develop robots to treat and test Covid-19 patients in a bid to protect health workers, The Telegraph, 2020.

11. W. Qi.China develops intelligent robots for throat swab sampling of coronavirus tests, Global Times, 2020.

12. A. Sharp, K. Kruusamae, B. Ebersole, and M. Pryor.Semiautonomous dual-arm mobile manipulator system with intuitive supervisory user interfaces, in Proc. IEEE Workshop on Advanced Robotics and its Social Impacts, 2017, pp. 1-6.

13. D. Kruse, J. T. Wen, and R. J. Radke.A sensor-based dual-arm tele-robotic system, IEEE Trans. Autom. Sci. Eng., vol. 12, no. 1, pp. 4-18, 2015.

14. M. Shahbazi, A. S. Farokh, C. Ward, H. A. Talebi, and R. V. Patel.Multimodal sensorimotor integration for expert-in-the-loop telerobotic surgical training, IEEE Trans. Robot., vol. 34, no. 6, pp. 1549-1564, 2018.

15. C. Y. Weng, W. C. Tan, and I. M. Chen.A survey of dual-arm robotic issues on assembly tasks, in CISM International Centre for Mechanical Sciences, Courses and Lectures, 2019, pp. 474-480.

16. X. Wang and L. Chen.A vision-based coordinated motion scheme for dual-arm robots, J. Intell. Robot. Syst. Theory Appl., vol. 97, no. 1, pp. 67-79, 2020.

17. R. Sakai et al.A mobile dual-arm manipulation robot system for stocking and disposing of items in a convenience store by using universal vacuum grippers for grasping items, $A d v$. Robot., 2019.

18. L. Shi, S. Kayastha, and J. Katupitiya.Robust coordinated control of a dual-arm space robot, Acta Astronaut., vol. 138, pp. 475-489, 2017.

19. I. V. Krechetov, A. A. Skvortsov, P. S. Lavrikov, and V. Korotkovs.Development of dual-arm assistant robot: Motion trajectory planning for the manipulator arm, Int. Rev. Mech. Eng., vol. 11, no. 9, pp. 683-691, 2017.

20. X. Ling, Y. Zhao, L. Gong, C. Liu, and T. Wang.Dual-arm cooperation and implementing for robotic harvesting tomato using binocular vision, Rob. Auton. Syst., vol. 114, pp. 134-143, 2019. https://doi.org/10.1016/j.robot.2019.01.019

21. E. M. Boctor, G. Fisher, M. A. Choti, G. Fichtinger, and H. Taylor.A dual-armed robotic system for intraoperative ultrasound guided hepatic ablative therapy: A prospective study, in Proc. 2004 IEEE International Coference on Roboticic \& Automation, 2004, pp. 2517-2522.

22. Q. Wu, M. Li, X. Qi, Y. Hu, B. Li, and J. Zhang.Coordinated control of a dual-arm robot for surgical instrument sorting tasks, Rob. Auton. Syst., vol. 112, pp. 1-12, 2019.

23. E. Franco et al.3D ultrasound-guided robotic steering of a flexible needle via visual servoing, Int. J. Comput. Assist. Radiol. Surg., vol. 15, no. 1, pp. 65-72, 2020.

24. Robotis.OpenMANIPULATOR-X, 2020. [Online]. Available:

https://emanual.robotis.com/docs/en/platform/openmani pulator_x/overview/. [Accessed: 02-Jul-2020].

25. Robotis.TurtleBot3, 2020. [Online]. Available: https://emanual.robotis.com/docs/en/platform/turtlebot3 /overview/.

26. K. A. Khan, R. R. Konda, and J.-C. Ryu.ROS-based control for a robot manipulator with a demonstration of the ball-on-plate task, $A d v$. Robot. Res., vol. 2, no. 2 , pp. 113-127, 2018.

27. S. Hernandez-Mendez, C. Maldonado-Mendez, A. Marin-Hernandez, H. V. Rios-Figueroa, H. Vazquez-Leal, and E. R. Palacios-Hernandez.Design and implementation of a robotic arm using ROS and MoveIt!, in 2017 IEEE International Autumn Meeting on Power, Electronics and Computing, ROPEC 2017, 2018, pp. 1-6. 